\title{
Komplexe Rekonstruktionen bei Charcot-Arthropathie mittels Ilizarov-Ringfixateur
}

\author{
Wirth, S H ; Espinosa, N ; Berli, M ; Jankauskas, L
}

\begin{abstract}
BACKGROUND: From the foot surgeon's point of view, the surgical treatment of Charcot neuro-osteopathy is considered challenging. Conservative treatment using plaster and orthoses is used to treat Charcot arthropathy, especially in the acute stage of a treatment strategy. Surgical treatment using the Ilizarov ring fixator is established for the correction of residual misalignments and also in the acute stage. Advantages of the ring fixator are great stability of the apparatus, low loading of the soft tissues caused by foreign material, and possible long-term treatment. OBJECTIVES: The aim of this work is to explain the indication, planning of correction, assembly of Ilizarov ring fixator, and the postoperative treatment with respect to the literature and the authors' experience. The aim of treatment with the Ilizarov ring fixator is a plantigrade, resilient, ulcer-free foot. Treatment is lengthy for both the affected patients and the attending orthopedic surgeon, and great attention is required to achieve the desired corrections and a good result for the patient. DISCUSSION: According to the literature and the authors' experience, the results indicate that the Ilizarov ring fixator represents a viable method for the preservation of the affected limb in patients with Charcot neuro-osteopathy. The assembly of the apparatus must follow the principles of Ilizarov in order to avoid failure. Corrective osseous and soft tissue interventions require a detailed preoperative analysis. Hintergrund: Die chirurgische Behandlung der Charcot-Neuroosteopathie ist aus fußchirurgischer Sicht als anspruchsvoll zu beurteilen. Die konservative Therapie mittels Gips- und Orthesenbehandlung ist in der Behandlung der CharcotArthropathie insbesondere im Akutstadium eine Behandlungsstrategie. Die chirurgische Behandlung mittels Ilizarov-Ringfixateur ist zur Korrektur der residuellen Fehlstellungen aber auch im Akutstadium als etabliert anzusehen. Die Vorteile des Ringfixateurs sind die große Stabilität des Apparats, die geringe Belastung der Weichteile durch Fremdmaterial und die mögliche Langzeitbehandlung. Ziel der Arbeit: Ziel der Arbeit ist es, die Indikationsstellung, Planung der Korrektur, Montage des Ilizarov-Ringfixateurs sowie die postoperative Nachbehandlung bezüglich Literatur und aus Sicht der Autoren zu erläutern. Angestrebt wird bei der Behandlung mit Ilizarov-Ringfixateur ein plantigrader, belastbarer, ulkusfreier Fuß. Die Nachbehandlung ist für die betroffenen Patienten als auch den behandelnden Orthopäden langwierig und bedarf großer Aufmerksamkeit, um die Umsetzung der angestrebten Korrekturen und ein gutes Resultat für den Patienten zu erreichen. Diskussion: Gemäß der Literatur als auch der Erfahrung der Autoren zeigen die Ergebnisse, dass die Korrektur mittels Ilizarov-Ringfixateur bei Patienten mit Charcot-Neuroosteopathie eine valable Methode zur Erhaltung der betroffenen Extremität darstellt. Die Montage des Apparats muss den Prinzipien von Ilizarov folgen, um ein Fehlschlagen der Intervention zu vermeiden. Die korrektiven ossären und weichteiligen Eingriffe bedürfen einer genauen Analyse der präoperativen Fehlstellung.
\end{abstract}

DOI: https://doi.org/10.1007/s00132-014-3063-y

Other titles: Complex reconstruction in Charcot arthropathy using the Ilizarov ring fixator 
ZORA URL: https://doi.org/10.5167/uzh-104626

Journal Article

Published Version

Originally published at:

Wirth, S H; Espinosa, N; Berli, M; Jankauskas, L (2015). Komplexe Rekonstruktionen bei CharcotArthropathie mittels Ilizarov-Ringfixateur. Der Orthopäde, 44(1):50-57.

DOI: https://doi.org/10.1007/s00132-014-3063-y 
Orthopäde 2015 • 44:50-57

DOI 10.1007/s00132-014-3063-y

Online publiziert: 17. Dezember 2014

c) Springer-Verlag Berlin Heidelberg 2014
S.H. Wirth ${ }^{1}$ N. Espinosa ${ }^{1}$ - M. Berli ${ }^{1}$ L. Jankauskas ${ }^{2}$

${ }^{1}$ Fuß- und Sprunggelenkchirurgie, Universitätsklinik Balgrist, Zürich, Schweiz

${ }^{2}$ Orthopädische Klinik Luzern AG, Luzern, Schweiz
Die Charcot-Arthropathie des Fußes stellt aus fußchirurgischer Sicht ein komplexes, schwer zu behandelndes Problem dar [1, 2]. Die frühzeitige Erkennung der Erkrankung ist wegweisend für den späteren Behandlungserfolg [3].

In der frühen Phase der Erkrankung bietet die konservative Therapie eine valable Option, den Verlauf der Krankheit zu verlangsamen und potentielle Deformitäten in ihrer Entwicklung aufzuhalten oder zumindest zu verzögern [4-8]. Ein Vorteil einer frühen operativen Korrektur scheint ein besser erhaltener Knochenstock zu sein, welcher die anatomische Rekonstruktion erlaubt. Ebenso zeigen sich Hinweise, dass die Heilungsphase deutlich verkürzt scheint [9]. Dennoch gibt es unseren Wissens nach in der Literatur derzeit keine Arbeiten, die prospektiv und randomisiert das konservative Vorgehen mit der operativen Korrektur oder Amputation vergleichen [2].

Ziel der operativen Therapie ist die im besten Fall physiologische Ausrichtung des Fußes, so dass ein plantigrader, stabiler Fuß, welcher mit Schuhen adäquat versorgt werden kann, resultiert $[9,10]$. Weitere Ziele der operativen Therapie sind die Infektfreiheit sowie die Vermeidung von Ulzera [10-12] und letztlich die Vermeidung einer Amputation $[9,10]$.

\section{Indikation}

Die Indikation zur Arthrodese mittels Ilizarov-Ringfixateur wird durch die chronische Neurosteoarthropathie bestimmt. In der akuten Phase ist zum einen die Osteolyse wegweisend, die durch die Destruk- tion des Knochens zu Frakturen und Luxationen führt, welche den Halt interner Fixationen deutlich herabsetzen ([13-15]; - Abb. 1a-c).

Der Weichteilmantel spielt eine wichtige Rolle. Sind bereits Ulzerationen vorhanden, ist eine Wundkontrolle bei Gipstherapie erschwert. Prekäre Hautverhältnisse, die einer Belastung und den damit assoziierten Scherkräften nicht gewachsen sind [15], verhindern eine adäquate Gipstherapie $[1,13]$.

\section{》) Das Patientengut ist oft übergewichtig und weist eine reduzierte Infektabwehr auf}

Schwere, instabile, nicht durch Gipsbehandlung oder orthopädietechnische Hilfsmittel adressierbare Fehlstellungen stellen eine weitere Indikation dar. Hier ist initial ebenfalls eine redressierende Gipsbehandlung indiziert, jedoch besteht bei bleibenden Fehlstellungen das erhöhte Risiko tiefer Ulzerationen und chronischer Osteomyelitiden [15, 16]. Gemäß der Literatur ist das Patientengut oft übergewichtig und weist eine reduzierte Infektabwehr auf. Somit ist bei diesen Patienten das Risiko für chirurgisch assoziierte Wundinfekte erhöht und indiziert ein möglichst weichteilschonendes Vorgehen [15, 17].

Indikationen zur operativen Versorgung mit dem Ilizarov-Ringfixateur:

- schlechte Knochenqualität,

- Infektrisiko,

- fehlende Compliance des Patienten,

- Entlastung erschwert,

- nicht gipsbare Fehlstellung des Mittelund/oder Rückfußes mit Ulzeration,
- nicht gipsbare Instabilität des Mittelund/oder Rückfußes mit Ulzeration,

- Revision mit schlechtem Knochenstock,

- schlechte Weichteilverhältnisse,

- verlängerte Knochenheilung.

\section{Vorteile des \\ Ilizarov-Ringfixateurs}

Der Ringfixateur bietet eine hohe biomechanische Stabilität [18]. Die Fixation auf mehreren Ebenen erlaubt eine multiplanare Kompression der verschiedenen Arthrodesezonen. Es resultiert eine hohe Rigidität des Konstrukts und ermöglicht eine frühe Belastung der betroffenen Extremität. Hierbei bleibt der $\mathrm{Zu}$ gang zu den Weichteilen erhalten und Wundkontrollen sind zu jederzeit möglich (• Abb. 2f-h, 3a-c).

Ein weiterer Vorteil ist die postoperative Anpassbarkeit. Durch die Eigenschaften des Apparats sind eine Anpassung der Kompression, eine Veränderung der Rotation sowie eine Verschiebung der Fragmente im postoperativen Verlauf möglich. Insbesondere bei chronischen Verläufen oder bei massiven Fehlstellungen ist der Weichteilmantel für eine einzeitige Korrektur zu empfindlich. Hier bietet die Ilizarov-Technik die Möglichkeit der schrittweisen Korrektur [19].

Bei Infektionen ist die interne Fixation kontraindiziert. Die Ilizarov-Technik erlaubt die Resektion der infizierten knöchernen und weichteiligen Strukturen und bietet genügend Stabilität, um die Infektion ausheilen zu lassen und weitere zu verhindern [1].

Bei Patienten mit diabetischer Angiopathie und herabgesetztem Wundhei- 

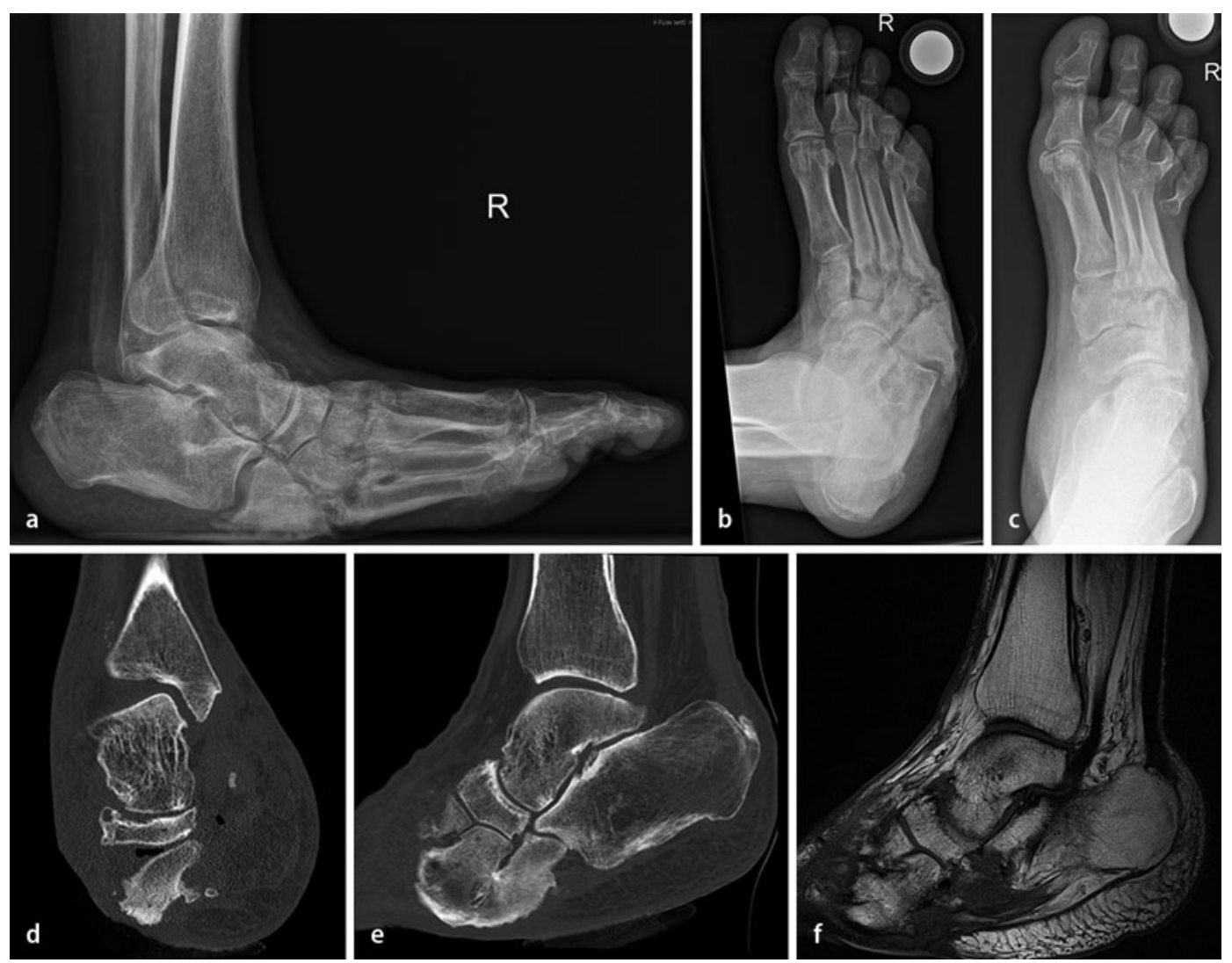

Abb. $1<$ a-c Einbruch des Fußgewölbes mit, "rocker bottom", schwere Ulzeration mit Osteomyelitis am lateralen Fußrand (Os cuboideum). Laterale Instabilität bei Status nach Resektion Os metatarsale $V$ rechts. d, e Computertomographie des Rückfußes mit Zerstörung des Os cuboideums und Lufteinschlüssen im Chopart-Gelenk. f Osteomyelitis Os cuboideum mit Weiteilulzeration

lungspotential bietet die externe Fixation mittels minimal-invasivem, perkutanem Zugang einen deutlichen Vorteil [1,9].

\section{》) Die llizarov-Technik bietet die Möglichkeit der schrittweisen Korrektur}

Durch die Konstruktion der Fine-wireFixateure ist eine lange Stabilität gewährleistet. Im Gegensatz zu herkömmlichen Fixateuren mit Schanz-Schraubenfixation bleibt der Ringfixateur bei korrekter Anwendung über Monate stabil. Dies ist insbesondere bei der Behandlung der Charcot-Arthropathie ein Vorteil, da die Behandlung bis zum Erreichen einer stabilen Arthrodese respektive stabilen Pseudarthrose 3 bis 6 Monate (in seltenen Fällen länger) dauern kann. Kommt es bei der Belastung zu einem Drahtbruch, so kann der Draht problemlos ausgewechselt werden.

Vorteile des Ilizarov-Fixateurs:

- biomechanische Stabilität,

- Zugang zu den Weichteilen bleibt erhalten,

- postoperative Anpassbarkeit,
- Stabilität bleibt lange konstant,

- bei akuten und chronischen Infektionen einsetzbar,

- weichteilschonend.

\section{Präoperative Vorbereitungen}

Die präoperative Planung ist bei komplexen Fehlstellungen wegweisend. Folgende Fragen müssen beantwortet werden:

1. Welcher Zugang ist am besten geeignet? Der Zugang sollte die Angiosomen respektieren und im Fall einer größeren Korrektur auf der konvexen Seite der Deformität liegen. Im Idealfall können Ulzera gleichzeitig exzidieren werden und die Weichteile primär geschlossen werden.

2. Liegt eine Osteomyelitis vor? Wenn ja, sollte die Resektion des infizierten Knochens in die Planung integriert werden.

3. Ausmaß und Lokalisation der Korrekturosteotomie/Arthrodese? Die Deformitäten werden bei CharcotArthropathie möglichst am Ort der Hauptfehlstellung korrigiert.

4. Wie wird der Ringfixateur montiert? Zuerst wird festgelegt, welche Fuß-/
Unterschenkelanteile mit dem Ringfixateur komprimiert werden müssen. Dann erfolgt die Größenbestimmung der Ringe/Halbringe sowie der Fußplatte. Anschließend ist die Position der Drähte zu überlegen. Zwei gekreuzte Drähte bilden zusammen mit einem damit verbundenen Ring/ Halbring einen Fixationspunkt. Anschließend sollten diese Fixationspunkte so verbunden werden, dass eine Kompression der gewünschten Arthrodesezone und in geplanter Richtung ermöglicht wird.

Eine radiologische Abklärung ist neben der klinischen Untersuchung von entscheidender Bedeutung. Eine konventionelle Bildgebung unter Belastung ist unersetzlich. Eine Computertomographie (CT) zur dreidimensionalen Analyse kann bei komplexen Deformitäten wertvoll sein. Ebenso gewinnt die Magnetresonanztomographie (MRT) zur Beurteilung der Weichteile und zum Nachweis einer Osteomyelitis einen immer höheren Stellenwert, wenngleich im akuten Stadium die Unterscheidung der typischen Char- 
cot-Veränderungen zu einer Osteomyelitis nicht immer gut gelingt $[6,20]$.

\section{》) Eine radiologische \\ Abklärung ist neben der klinischen Untersuchung von entscheidender Bedeutung}

Die Planung der Korrektur und der Fixationspunkte mittels Schemazeichnungen hat sich bewährt (• Abb. 4). Bei der Planung ist darauf zu achten, dass die zu errechnende Korrektur mit möglichst einfachen Mitteln zu erreichen ist.

Die bereits präoperative Montage des Ringfixateurs und Sterilisation des montierten Ringfixateurs wird vielerorts praktiziert und ist ebenfalls mit einer intraoperativen Zeitersparnis verbunden. Jedoch kann im Falle einer Fehlinterpretation der intraoperativen Situation dieses Vorgehen zu einem größeren Zeitverlust führen und wird deshalb von den Autoren nicht praktiziert.

\section{Operatives Vorgehen}

\section{Korrektur der Fehlstellung}

Der Patient ist in Rückenlagerung gelagert. Für die ersten Schritte wird eine Blutsperre montiert und aktiviert. Die Blutleerezeit darf eine maximale Dauer von $2 \mathrm{~h}$ nicht überschreiten. Nach der provisorischen Fixation der Arthrodese/ Osteotomie mit Kirschner-Drähten wird die Blutsperre eröffnet. Falls ein infiziertes Ulkus vorliegt (respektive Osteomyelitis) und der Erreger bekannt ist, wird vor Schnitt eine resistenzgerechte Antibiose verabreicht. Es empfiehlt sich, Ulzera und infizierten Knochen in einem ersten Schritt $\mathrm{zu}$ debridieren und anschließend das benutzte Instrumentarium abzugeben und neu abzudecken. Eine bakteriologische Untersuchung des gewonnen Gewebes ist auch bei bekanntem Keim essentiell, um zusätzliche Erreger zu identifizieren.

\section{Korrigierende OSG-Arthrodese}

Das obere Sprunggelenk (OSG, Typ 3A, Brodsky [21]) ist von der Charcot-Arthropathie relativ selten betroffen (19\% der Fälle [21]). Die Kontraktur der Achillessehne ist eine wichtige Komponente der Fehlstellung und muss zuerst angegangen

Orthopäde 2015 - 44:50-57 DOI 10.1007/s00132-014-3063-y

(c) Springer-Verlag Berlin Heidelberg 2014

\section{S.H. Wirth · N. Espinosa - M. Berli $\cdot$ L. Jankauskas}

Komplexe Rekonstruktionen bei CharcotArthropathie mittels Ilizarov-Ringfixateur

\section{Zusammenfassung}

Hintergrund. Die chirurgische Behandlung der Charcot-Neuroosteopathie ist aus fußchirurgischer Sicht als anspruchsvoll zu beurteilen. Die konservative Therapie mittels Gipsund Orthesenbehandlung ist in der Behandlung der Charcot-Arthropathie insbesondere im Akutstadium eine Behandlungsstrategie. Die chirurgische Behandlung mittels Ilizarov-Ringfixateur ist zur Korrektur der residuellen Fehlstellungen aber auch im Akutstadium als etabliert anzusehen. Die Vorteile des Ringfixateurs sind die große Stabilität des Apparats, die geringe Belastung der Weichteile durch Fremdmaterial und die mögliche Langzeitbehandlung.

Ziel der Arbeit. Ziel der Arbeit ist es, die Indikationsstellung, Planung der Korrektur, Montage des llizarov-Ringfixateurs sowie die postoperative Nachbehandlung bezüglich Literatur und aus Sicht der Autoren zu erläutern. Angestrebt wird bei der Behandlung mit Ilizarov-Ringfixateur ein plantigrader, belastbarer, ulkusfreier Fuß. Die Nachbehandlung ist für die betroffenen Patienten als auch den behandelnden Orthopäden langwierig und bedarf großer Aufmerksamkeit, um die Umsetzung der angestrebten Korrekturen und ein gutes Resultat für den Patienten zu erreichen.

Diskussion. Gemäß der Literatur als auch der Erfahrung der Autoren zeigen die Ergebnisse, dass die Korrektur mittels Ilizarov-Ringfixateur bei Patienten mit Charcot-Neuroosteopathie eine valable Methode zur Erhaltung der betroffenen Extremität darstellt. Die Montage des Apparats muss den Prinzipien von Ilizarov folgen, um ein Fehlschlagen der Intervention zu vermeiden. Die korrektiven ossären und weichteiligen Eingriffe bedürfen einer genauen Analyse der präoperativen Fehlstellung.

\section{Schlüsselwörter}

Korrekturosteotomie · Rückfuß · Weichteile · Ringfixateur $\cdot$ Arthrodese

\section{Complex reconstruction in Charcot arthropathy using the llizarov ring fixator}

\section{Abstract}

Background. From the foot surgeon's point of view, the surgical treatment of Charcot neuro-osteopathy is considered challenging. Conservative treatment using plaster and orthoses is used to treat Charcot arthropathy, especially in the acute stage of a treatment strategy. Surgical treatment using the llizarov ring fixator is established for the correction of residual misalignments and also in the acute stage. Advantages of the ring fixator are great stability of the apparatus, low loading of the soft tissues caused by foreign material, and possible long-term treatment.

Objectives. The aim of this work is to explain the indication, planning of correction, assembly of llizarov ring fixator, and the postoperative treatment with respect to the literature and the authors' experience. The aim of treatment with the llizarov ring fixator is a plan- tigrade, resilient, ulcer-free foot. Treatment is lengthy for both the affected patients and the attending orthopedic surgeon, and great attention is required to achieve the desired corrections and a good result for the patient. Discussion. According to the literature and the authors' experience, the results indicate that the llizarov ring fixator represents a viable method for the preservation of the affected limb in patients with Charcot neuro-osteopathy. The assembly of the apparatus must follow the principles of Ilizarov in order to avoid failure. Corrective osseous and soft tissue interventions require a detailed preoperative analysis.

Keywords

Osteotomy - Foot bones - Soft tissue injuries . Fixation devices, external $\cdot$ Arthrodesis werden. In unserer Praxis hat sich die minimal-invasive Technik mittels 3 Stichinzisionen im Abstand von jeweils 2-3 cm bewährt. Ist dies zur Reduktion der Fehlstellung nicht ausreichend, stellt die Tenotomie der Achillessehne eine Option dar.
Bei einer Varusfehlstellung ist ein klassischer lateraler transfibulärer $\mathrm{Zu}$ gang am besten geeignet. Der destruierte Knochen wird sowohl an der Tibia wie auch am Talus senkrecht zur Längsachse des Unterschenkels mit einer oszillie- 


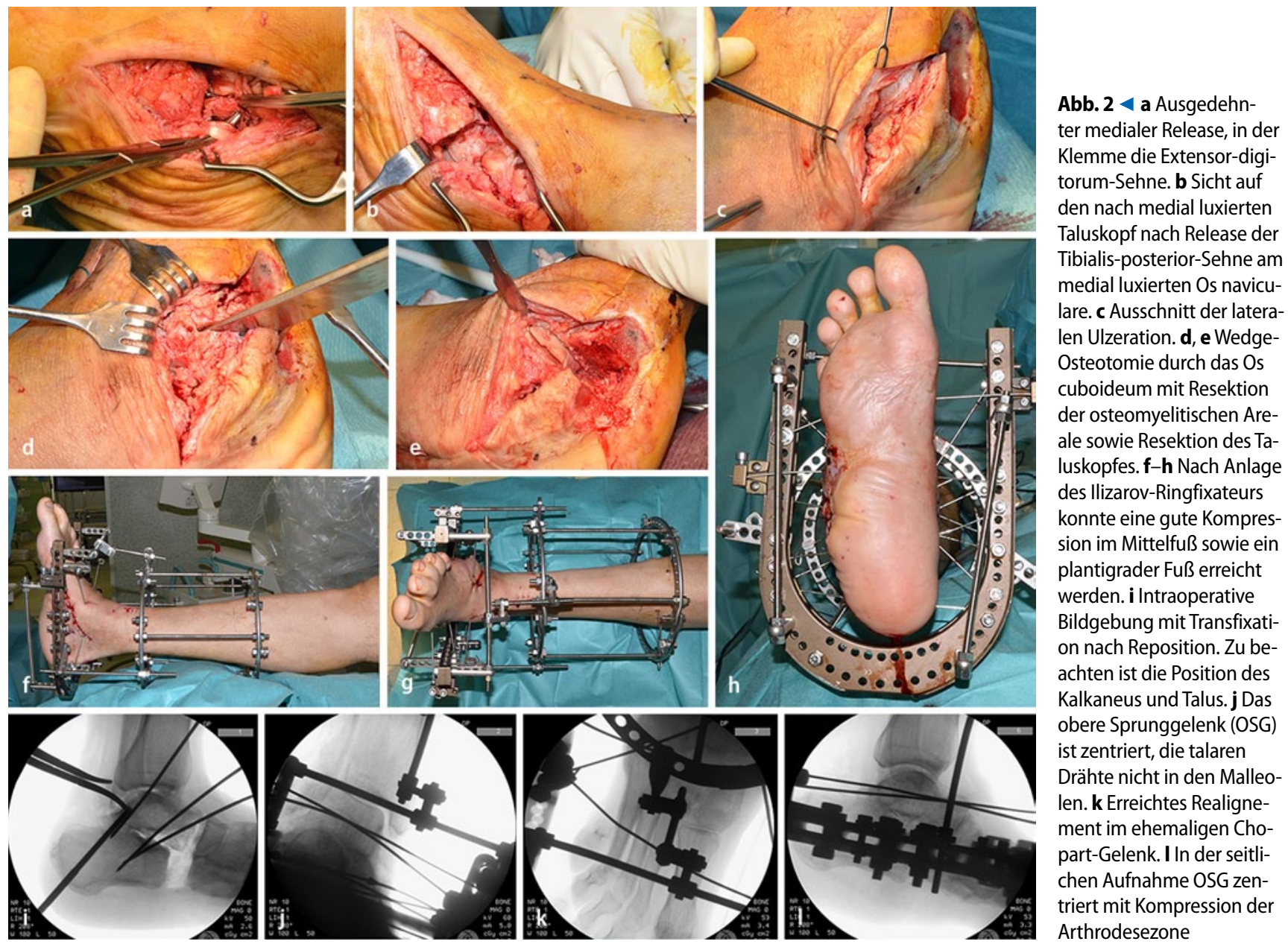

renden Säge reseziert. Liegt eine ausgedehnte Destruktion der Tibia vor, kann eine Osteotomie des Malleolus medialis durch einen kleinen medialen Zugang nötig sein. Auch das distale Fibulafragment muss häufig proximal großzügig gekürzt werden. Dann wird der Fuß in plantigrader Stellung und korrekter Rotation an die Tibia mit 2-3 KirschnerDrähten ( $2 \mathrm{~mm}$ Dicke) provisorisch fixiert (• Abb. 2i). Unter Bildverstärker(BV-)Kontrolle wird die Stellung geprüft. Die anschließende Montage des Ringfixateurs soll die Kompression der Tibia gegen Talus gewährleisten.

Liegt eine ausgedehnte Destruktion des Taluskörpers vor, kann dieser reseziert und eine tibiokalkaneare Arthrodese mit Hilfe des Ringfixateurs angestrebt werden.

\section{Korrigierende subtalare Arthrodese} Der subtalare Komplex (Typ 2, Brodsky [21]) ist in etwa $28 \%$ der Fälle betroffen [21]. In Abhängigkeit der Dominanz der
Destruktion kann sich sowohl ein KnickSenkfuß (Destruktion kalkaneokuboidal) oder eine Adduktusdeformität (Destruktion talonavikular) entwickeln. Das muskuläre Ungleichgewicht der Invertoren/Evertoren infolge der Polyneuropathie spielt eine wichtige Rolle. Eine iatrogene Läsion der Peronealsehnen in Folge Resektion der Basis des Metatarsale V bei einer „Exostosektomie“ kann ebenfalls zur Adduktus- und Varusdeformität führen (• Abb. 1).

$>$ Eine Kontraktur der Achillessehne ist in der Mehrzahl der Fälle vorhanden.

Nach einer Verlängerung der Achillessehne wird die eigentliche Deformität korrigiert. Die Darstellung des unteren Sprunggelenks (USG) erfolgt durch einen lateralen Zugang subfibulär. Mobilisierung des USG und sorgfältige Entknorpelung sind essentiell. Bei der Reposition sollte beachtet werden, die "gesunde" Seite durch eine zuklappende Os- teotomie am Chopart-Gelenk zu verkürzen, d. h. bei einer Außenrotation des Vorfußes muss die zuklappende Osteotomie medial, bei einer Adduktusdeformität lateral durchgeführt werden.

Bei einer Adduktusdeformität müssen häufig die kontrakten Invertoren des Fußes verlängert oder in bestimmten Fällen tenotomiert werden. Ausnahme bildet die Sehne des M. tibialis anterior. Trägt diese Sehne wesentlich zur Adduktusdeformität bei, muss sie auf das Os cuboideum transferiert werden.

Nach dem Release der Invertoren, des USG und der zuklappenden Osteotomie auf der konvexen Seite ist in der Regel eine korrigierende subtalare (talokalkanear und im Chopart-Gelenk) Arthrodese möglich. Nach der provisorischen Fixation mit dicken Kirschner-Drähten wird die Stellung unter BV kontrolliert und der Ringfixateur angelegt. Der montierte Ringfixateur soll eine Kompression sowohl im Chopart-Gelenk in der Längsachse des Fußes wie auch zwischen Ta- 

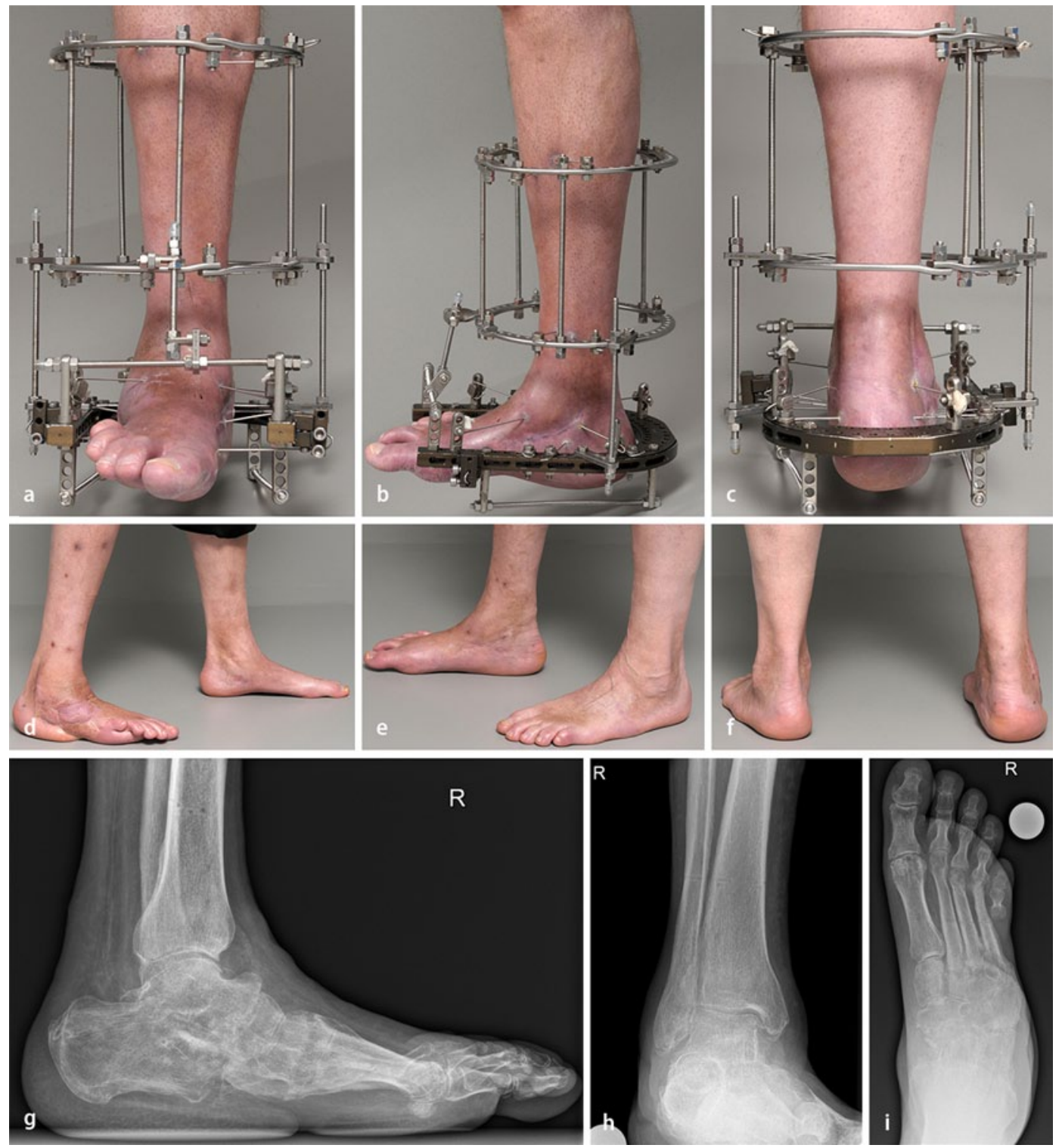

Abb. $3<$ a-c Stabiler Fixateur 3 Monate postoperativ, gute Weichteilverhältnisse, gutes Alignement der Rückfußachse. In der Röntgenkontrolle zeigte sich eine zunehmende Konsolidation der Arthrodese. d-f 6 Monate postoperativ, nach Entfernung des Ilizarov-Ringfixateurs, plantigrader Fuß mit korrigierter Rückfußachse, Weichteile reizlos. g-i Röntgenkontrolle 6 Monate postoperativ zeigt Konsolidierung der Arthrodesen im Rückfuß und Chopart-Gelenk. Keine ossären Druckpunkte plantar in der seitlichen Projektion, oberes Sprunggelenk zentriert

lus und Kalkaneus in der Längsachse des Unterschenkels ermöglichen.

\section{Korrigierende Mittelfußosteotomie}

In $60-70 \%$ der Fälle liegt eine Beteiligung des Mittelfußes vor (Typ 1, Brodsky [21]). Hierbei kommt es zu einem Kollaps des Mittelfußes, welcher in einer plantaren Prominenz der lateralen knöchernen Strukturen (kalkaneokuboidales Gelenk) mit Ulzerationen resultiert [1]. Häufig liegt eine Osteomyelitis des Os cuboideum vor (• Abb. 1f). Der Rückfuß ist meistens in Equinusfehlstellung, wobei der Vorfuß in der Dorsalextension und Außenrotation fixiert ist. So kommt es zu der sog. Tintenlöscherdeformität (• Abb. 1a).
Die Korrektur der Equinusfehlstellung im Rückfuß macht auch in diesen Fällen eine Verlängerung der Achillessehne notwendig. Der nächste Schritt ist die Korrekturosteotomie des Mittelfußes. Eine anatomische Rekonstruktion der destruierten und luxierten Fußwurzel nach Prinzipien der elektiven, „klassischen " Fußchirurgie ist in den meisten Fällen nicht möglich. Die medial/plantar zuklappende Korrekturosteotomie des Mittelfußes am Ort der Deformität ist gemäß unserer Erfahrung eine potente Korrekturmöglichkeit. Der Zugang ist medial gelegen. Die plantaren Strukturen werden mit einem großen Hohmann-Haken geschützt. Die Osteotomie erfolgt mit einem 10-mm-Meißel. Die Größe des Keils richtet sich nach dem Ausmaß der Abduktion und Dorsalextension. Die Osteotomie erfolgt biplanar. Gelegentlich ist ein kleiner lateraler Zugang notwendig, um die Osteotomie besser kontrollieren zu können. Die komplette Mittelfußosteotomie ermöglicht die Derotation des Vorfußes.

Nach provisorischer Fixation mit 2 Kirschner-Drähten und anschließender BV-Kontrolle wird ein Ringfixateur angelegt. Eine Kompression zwischen Rückund Vorfuß muss gewährleistet werden, wobei eine leichte Plantarflexion im Mittelfuß erwünscht wird.

\section{Komplexe Rückfuß- und Mittelfußrekonstruktion}

In komplexen Fällen arbeitet man nach den gleichen Regel, nur dass der Aufbau des Ringfixateurs deutlich komplizierter 

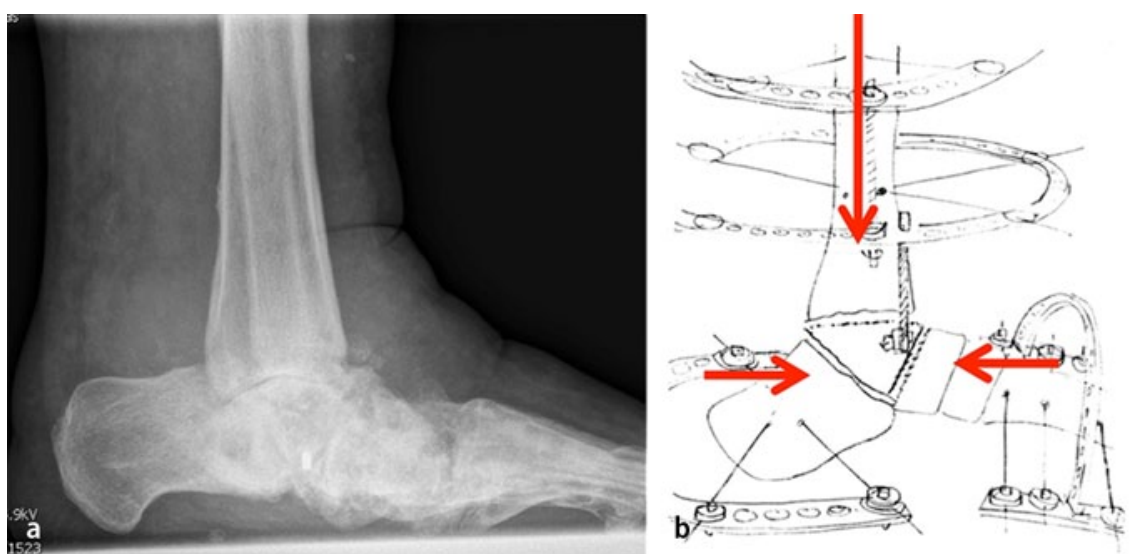

Abb. 4 ム Planung der Rekonstruktion mit Hilfe des Röntgenbildes (a). Die Skizze (b) verdeutlicht die geplante Kompression (Pfeile) der Fragmente

wird, weil eine Kompression in mehreren Richtungen gewährleistet werden muss.

\section{Montage des llizarov-Ringfixateurs}

Die Montage des Ilizarov-Ringfixateurs erfordert die konsequente Einhaltung folgender Prinzipien:

1. Stabile Fixation des Ringfixateurs am Knochen,

2. Stabile Fixation der ossären Fragmente.

3. Die Montage muss die erwünschte geführte Bewegung (in diesem Fall Kompression) der ossären Fragmente ermöglichen.

Die Stabilität der Montage wird durch die Fixation am Unterschenkel gewährleistet. Unterschenkel, Rückfuß/Kalkaneus, Mittelfuß und Vorfuß werden als die separaten ossäre Fragmente betrachtet. Jedes Fragment benötigt idealerweise zwei Fixationspunkte, um eine stabile Situation zu erreichen. Ein Fixationspunkt besteht aus zwei gekreuzten Drähten, die eine Ebene bilden. Am Unterschenkel bildet ein Ring mit zwei gekreuzten Drähten einen Fixationspunkt. Zwei solche Ringe, verbunden mit 4 Gewindestäben, bilden einen Block. Ein Winkel nahe $90^{\circ}$ zwischen den Drähten sowie Olivendrähte erhöhen die Stabilität.

Im Bereich der Fußwurzel ist es in der Regel schwierig, 2 Fixationspunkte pro Fragment zu verwirklichen. Dort wird die Stabilität mit 2-3 gekreuzten Drähten erreicht.

Der Aufbau des Ringfixateurs beginnt proximal. Die Höhe des ersten Fi- xationspunktes ist auf Höhe des mittleren Drittels. Dort werden 2 gekreuzte Drähte in möglich großem Winkel zueinander eingebracht. Die Drähte sollten einen Durchmesser von 1,5 $\mathrm{mm}$ haben, da dickere Drähte den Ringfixateur zu rigide machen und dünnere Drähte zu leicht brechen [19]. Jeder Draht muss 2 Kortikalices durchbohren. Der erste Draht wird von posterolateral nach anteriomedial durch die Tibia eingebracht und ohne Olive gewählt. Die Ausrichtung des ersten Drahtes bestimmt die Ausrichtung des gesamten Ringfixateurs. Somit ist hier mit großer Sorgfalt vorzugehen. Der zweite Draht wird von posteromedial am ersten Ring gelegt und gespannt. Es empfiehlt sich nach der Fixation des Drahtes am ersten Ring den Block (2 Ringe, 2 Gewindestäben) zu montieren. Bei Fixation der Gewindestäbe richtet sich der zweite Ring parallel zum ersten aus und kann als Referenz für den zweiten Fixationspunkt benutzt werden. Der zweite Fixationspunkt ist das distale Drittel. Die Drähte werden in gleicher Weise wie oben beschrieben eingebracht, jedoch etwas flacher. Der laterale Draht darf die Fibula fassen.

Die Kenntnis der Anatomie der neurovaskulären Strukturen ist bei der Drahtanlage essentiell [19]. Eine lange Fußplatte hat sich für die Fixation der ossären Fragmente des Fußes in unseren Fällen bewährt.

Der Taluskörper wird mit gekreuzten Drähten von posteromedial retromalleolär (ventral der Sehne des M. tibialis posterior) und von posterolateral (zwischen
M. peronei und Achillessehne) fixiert (• Abb. 5a).

Der Talushals kann sowohl von medial wie auch von lateral mit Kirschner-Drähten gefasst werden. Der Kalkaneus kann mit 2 gekreuzten Drähten im Tuberbereich und mit einem dritten Draht längs von medial posterior nach distal lateral fixiert werden (• Abb. 5d).

Im Mittelfuß kann sowohl von medial wie auch von lateral ein Kirschner-Draht durch Os cuboideum und Ossa cuneiformia und 2 Drähte durch die Metatarsalia von medial und von lateral mit Oliven gelegt werden.

Die Kompression zwischen Tibia und Talus/Kalkaneus bei einer OSG-Arthrodese oder tibiokalkanearen Arthrodese wird durch Anziehen der Gewindestäbe zwischen dem Unterschenkelblock und der Fußplatte erreicht. Es ist zu bemerken, dass bei jeder Fußrekonstruktion sowohl Talus wie auch Kalkaneus und der Vorfuß an die Fußplatte fixiert werden müssen, da sonst die Stabilität der Konstruktion ungenügend ist.

Bei einer subtalaren Arthrodese oder Mittelfußrekonstruktion ist es meist nicht möglich, eine klassische Kompression zwischen den Blöcken/Ringen durchzuführen. Hierfür müssen die Drähte vorgebogen werden ( $\bullet$ Abb. 5b). Durch Anbringen von Spannung an den Drähten wird dann durch die Vorbiegung der Drähte Druck auf die subtalare Arthrodesezone oder auch im Mittelfußbereich ausgeübt (•Abb. 5c).

\section{Fallbeispiel}

In dieser Kasuistik handelt es sich um einen 47-jährigen Patienten mit CharcotArthropathie. Aufgrund von Ulzerationen wurde das Os metatarsale $V$ entfernt (• Abb. 1a-c). Es zeigte sich erneut eine tiefe Ulzeration am lateralen Fußrand. Eine Knochenprobe war positiv und es stellte sich ein hochgradiger Verdacht auf eine Osteomyelitis des Os cuboideum ein. Klinisch ergab sich eine deutliche Equinovarusfehlstellung.

Die CT zeigte Lufteinschlüsse im Kalkaneokuboidalgelenk und eine Zerstörung des Os cuboideum ( $\bullet$ Abb. 1d, e), die MRT deutliche Hinweise auf eine Osteomyelitis des Os cuboideum ( $\bullet$ Abb. 1f). 


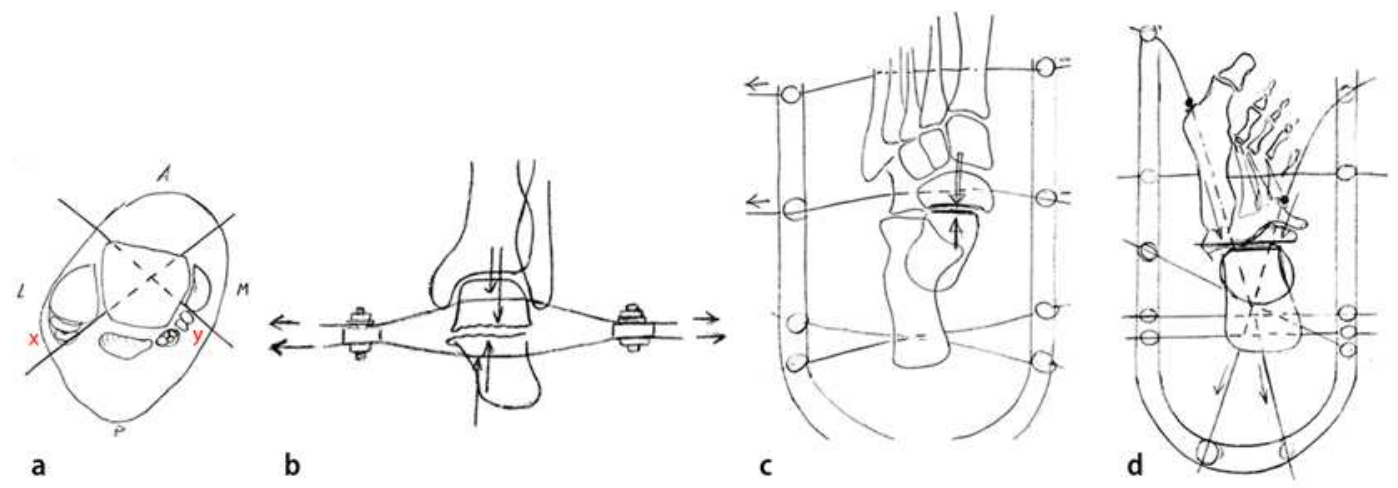

Abb. $5 \Delta$ a Lage der Drähte im Taluskörper ( $A$ anterior, $M$ medial, $P$ posterior, $L$ lateral, $x$ Position der Peronaeus-longus- und -brevis-Sehnen, $y$ Lage der A.-tibialis-posterior-, N.-tibialis-, M.-tibialis-posterior-Sehne). b Kompression subtalar durch Vorbiegen der Drähte. c Talonavikulare Kompression durch Vorbiegen der Drähte. d Lage der Drähte zur Unterstützung des axialen Druckes parallel zur Fußachse (zu beachten sind die Oliven distal)

Die Rekonstruktion wurde mittels Ilizarov-Ringfixateur unter Erhalt des OSG und Débridement der infizierten ossären Anteile mittels Wedge-Osteotomie vorgenommen (• Abb. 2a-e). Nach Montage des Ilizarov-Apparats konnte eine plantigrade Stellung des Fußes erreicht werden (- Abb. 2f-h). Die intraoperative Bildgebung ( $\bullet$ Abb. 2i-I) zeigte die Drahtlage und Kompression. Der postoperative Verlauf zeigte sich ereignislos.

Regelmäßige Kontrollen erfolgten initial 14-tägig, dann 3-wöchentlich. Der Ringfixateur war stets stabil, im Verlauf waren kein Kompressionsverlust, Pintrack-Infekt oder Lockerung festzustellen. 6 Wochen postoperativ zeigten sich deutlich gebesserte Weichteilverhältnisse und eine reizlose Wundheilung. Es bestand kein Hinweis auf eine Prolongation des Infekts (• Abb. 3a-c). Nach 6 Monaten konnte der Ringfixateur entfernt werden (• Abb. 3d, e).

Eine Röntgenkontrolle nach 6 Monaten ( $\bullet \mathbf{A b b} . \mathbf{3 g}-\mathbf{i})$ zeigte einen Durchbau der angestrebten Arthrodesen bei guter Stellung. Es folgte eine 6-wöchige Mobilisation im Unterschenkelgehgips mit schrittweiser Aufbelastung. Aktuell ist der Patient in orthopädischen Spezialschuhen mobil und seit 2 Jahren ulkusfrei.

\section{Postoperatives Management}

Die postoperative Betreuung der Patienten mit komplexer Rekonstruktion bei Charcot-Arthropathie ist für beide Seiten, den Patienten und die behandelnden Ärzte und Pflegenden aufwendig. Es han- delt sich um eine lange dauernde postoperative Phase. Nicht selten muss der Ringfixateur gerade bei komplexen Fällen für 4-6 Monate getragen werden. RegelmäBige Kontrollen sind in dieser Zeit absolut notwendig, um Lockerungen, Verschiebungen der Fragmente, Korrekturverlust, Wiederaufflammen von Infektionen, Pin-track-Infektionen etc. frühzeitig zu bemerken und zu beheben.

In der ersten postoperativen Phase steht die Wundheilung im Vordergrund.

Oftmals ist bei assoziierten Osteomyelitiden und prekären Hautverhältnissen eine VAC -Therapie („vacuum assisted closure") oder eine i.v.-Antibiotikatherapie notwendig. In der ersten Phase sind deshalb die Ruhigstellung zur Abschwellung der betroffenen Extremität und tägliche Wundkontrollen notwendig. Bei abgeheilten Wundverhältnissen ist eine ambulante Therapie denkbar. Es empfiehlt sich initial kurze Intervalle zu wählen, um oben erwähnte Komplikationen frühzeitig zu erkennen. Nach 6 Wochen sollte eine erste Röntgenkontrolle zur Beurteilung der Konsolidation, der Stellung und der intraossären Lage der Drähte stattfinden.

Bei guten Weichteilverhältnissen kann bei entsprechender Compliance des $\mathrm{Pa}$ tienten mit der Teilbelastung der betroffenen Extremität begonnen werden. Je nach Verlauf und Schwere des Falles ist eine Entfernung des Ringfixateurs ab dem 4. Monat postoperativ denkbar. In unserer Praxis sind nicht selten 6 Monate notwendig.
Nach Entfernung des Ringfixateurs ist eine Übergangsphase mit langsamem Belastungsaufbau im Unterschenkelgehgips sinnvoll. Diese Phase muss genutzt werden, um eine definitive orthopedietechnische Versorgung, wie Schuhe, Orthesen etc. in die Wege zu leiten. Auch nach Abschluss der Ringfixateurbehandlung ist eine regelmäßige Kontrolle im ersten Jahr alle 3 Monate und im Verlauf halbjährlich dringend zu empfehlen, um allfällige Rezidive, Probleme mit dem Schuhwerk und Ulzerationen zeitnah zu erfassen und zu behandeln.

\section{Fazit für die Praxis}

- Symptomatische, schwer kontrollierbare Deformitäten und Erkrankungen im Rahmen der Charcot-Neuroosteoarthropathie stellen hohe Anforderungen an den orthopädischen Chirurgen.

- Bleiben die primär adäquat durchgeführten konservativen Therapien erfolglos, müssen chirurgische Korrekturen erwogen werden. Ziel ist ein plantigrades, mit Hilfsmitteln gut einstellbares Fuß- und Sprunggelenk, welches eine Ambulation erlaubt. Hierfür eignet sich die Operation mittels extern angelegtem, die kranken Zonen überbrückendem Ringfixateur sehr gut.

- Eine präzise präoperative Planung und Abklärung, sowie rigorose Nachbehandlung bei diesem speziellen $\mathrm{Pa}$ tientengut ermöglicht die Umsetzung der angestrebten Korrekturen und ein gutes Resultat. 


\section{Korrespondenzadresse}

\section{Dr. S.H. Wirth}

Fuß- und Sprunggelenkchirurgie

Universitätsklinik Balgrist, Forchstraße 340

8008 Zürich

stephan.wirth@balgrist.ch

\section{Einhaltung ethischer Richtlinien}

Interessenkonflikt. S.H. Wirth, N. Espinosa, M. Berli und L. Jankauskas geben an, dass kein Interessenkonflikt besteht.

Dieser Beitrag enthält keine Studien an Menschen oder Tieren.

\section{Literatur}

1. Conway JD (2008) Charcot salvage of the foot and ankle using external fixation. Foot Ankle Clin 13(1):157-173. doi:10.1016/j.fcl.2007.12.002

2. Lowery NJ, Woods JB, Armstrong DG, Wukich DK (2012) Surgical management of Charcot neuroarthropathy of the foot and ankle: a systematic review. Foot Ankle Int 33(2):113-121. doi:10.3113/ FAl.2012.0113

3. Jolly GP, Zgonis T, Polyzois V (2003) External fixation in the management of Charcot neuroarthropathy. Clin Podiatr Med Surg 20(4):741-756. doi:10.1016/S0891-8422(03)00071-5
4. Pinzur MS, Lio T, Posner M (2006) Treatment of Eichenholtz stage I Charcot foot arthropathy with a weightbearing total contact cast. Foot Ankle Int 27(5):324-329

5. Pinzur MS (2008) Static ring fixation for nonplantigrade Charcot midfoot deformity. Oper Tech Orthop 18(4):287-292. doi:10.1053/j.oto.2009.01.006

6. Mittlmeier T, Klaue K, Haar P, Beck M (2008) Charcot foot. Current situation and outlook. Unfallchirurg 111(4):218-231. doi:10.1007/s00113-0081431-y

7. Zwipp H, Rammelt S, Dahlen C, Reichmann H (1999) Charcot foot. Orthopade 28(6):550-558

8. Frykberg RG (1991) The high risk foot in diabetes mellitus. Churchill Livingstone, New York

9. Cooper PS (2002) Application of external fixators for management of Charcot deformities of the foot and ankle. Foot Ankle Clin 7(1):207-254. doi:10.1016/S1083-7515(02)00019-0

10. Wukich DK, Belczyk RJ, Burns PR, Frykberg RG (2008) Complications encountered with circular ring fixation in persons with diabetes mellitus. Foot Ankle Int 29(10):994-1000. doi:10.3113/ FAl.2008.0994

11. Farber DC, Juliano PJ, Cavanagh PR (2002) Single stage correction with external fixation of the ulcerated foot in individuals with Charcot neuroarthropathy. Foot Ankle Int 23(2):130-134. doi:10.1177/107110070202300209

12. Zgonis T, Roukis TS, Lamm BM (2007) Charcot foot and ankle reconstruction: current thinking and surgical approaches. Clin Podiatr Med Surg 24(3):505-517. doi:10.1016/j.cpm.2007.03.004
13. Herbst SA (2004) External fixation of Charcot arthropathy. Foot Ankle Clin 9(3):595-609. doi:10.1016/j.fcl.2004.05.010

14. Petrova NL, Foster AVM, Edmonds ME (2005) Calcaneal bone mineral density in patients with Charcot neuropathic osteoarthropathy: differences between Type 1 and Type 2 diabetes. Diabet Med 22(6):756-761. doi:10.1111/j.14645491.2005.01510.x

15. Pinzur MS (2006) The role of ring external fixation in Charcot foot arthropathy. Foot Ankle Clin 11(4):837-847. doi:10.1016/j.fcl.2006.06.006

16. Papa J, Myerson M, Girard P (1993) Salvage, with arthrodesis, in intractable diabetic neuropathic arthropathy of the foot and ankle. J Bone Joint Surg Am 75(7):1056-1066

17. Herbst SA, Jones KB, Saltzman CL (2004) Pattern of diabetic neuropathic arthropathy associated with the peripheral bone mineral density. J Bone Joint Surg Br 86(3):378-383

18. Mullins MM, Davidson AW, Goodier D, Barry M (2003) The biomechanics of wire fixation in the llizarov system. Injury 34(2):155-157

19. Kirienko A, Villa A, Calhoun JH (2003) Ilizarov technique for complex foot and ankle deformities. Taylor \& Francis, Philadelphia

20. Pinzur MS (2007) Current concepts review: Charcot arthropathy of the foot and ankle. Foot Ankle Int 28(8):952-959. doi:10.3113/FAl.2007.0952

21. Brodsky JW (1992) Management of Charcot joints of the foot and ankle in diabetes. Semin Arthroplasty 3:58-62. doi:10.1111/os.12032/full

\section{Springer Medizin}

\section{Neu: Aktuelle Operationstechniken im Video Reichen Sie Ihren Beitrag für Der Orthopäde jetzt ein!}

Im Mittelpunkt des Beitrags steht Ihr Video zu aktuellen Operationstechniken. Es werden bewährte operative Verfahren dargestellt. Neben der Vermittlung aller wichtigen prä-, intraund postoperativen Details sowie den Hinweisen auf mögliche

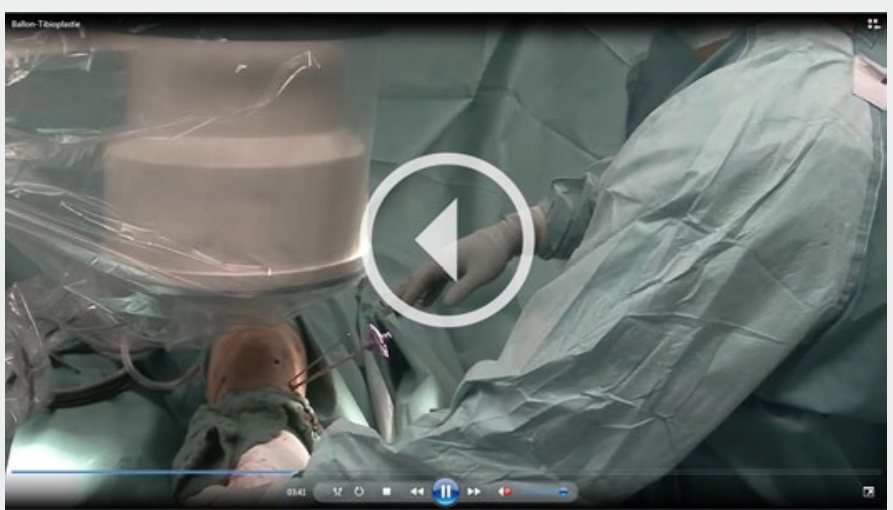

Alle Operationsschritte werden übersichtlich im Detail von Experten erklärt. Die neue Videoreihe finden Sie auf springermedizin.de
Fehler und Gefahren liegt der Schwerpunkt auf einer genauen Darstellung der jeweiligen Operationsschritte im Video. Nutzen Sie das Medium des Videos um komplizierte Vorgehensweisen leicht verständlich zu präsentieren. Bitte reichen Sie zudem einen kurzen begleitenden Beitrag ein, der die wesentlichen Punkte des Videos zusammenfasst. Das Copyright des Videos bleibt beim Autor.

Weitere Informationenzur Einreichung sowie einen detaillierten Autorenleitfaden finden Sie auf www.DerOrthopaede.de sowie editorialmanager.com/deor/ 